\title{
SOME PROBLEMS OF MATHEMATICS AND SCIENCE ${ }^{1}$
}

\author{
BY R. J. DUFFIN
}

Introduction. The development of mathematics has often been aided by the use of models from science and technology. There are three main reasons why models help: (i) attention is focused on significant problems; (ii) the intuition is aided in perceiving complex relations; (iii) new concepts are suggested. This paper describes problems arising from models which have interested me. The models come from physics, chemistry, engineering, and economics.

1. The Dirichlet problem for the wave equation. We are given two photographs of a vibrating string, one at time $t=0$ and another at a later time $t=\alpha$, as illustrated in Figure 1. Is it possible to determine the state

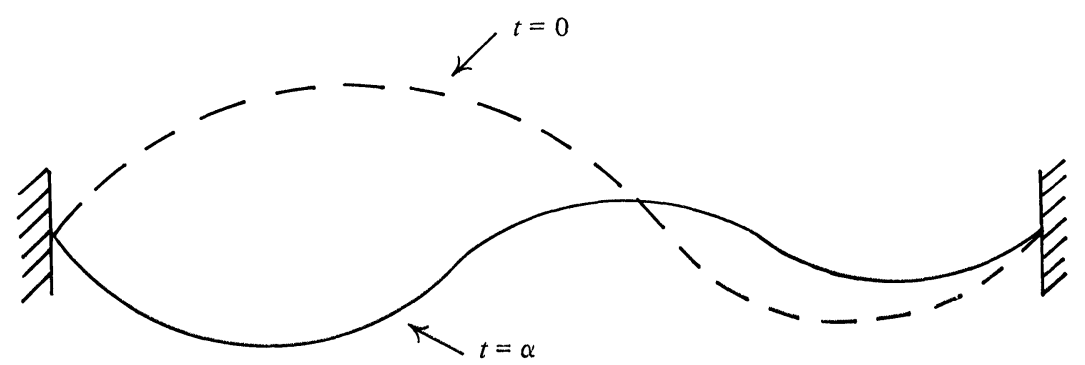

FIGURE 1. The vibrating string

at intermediate times? This puzzle led David Bourgin and me to study the Dirichlet problem for the wave equation. The wave equation is

$$
\partial^{2} y / \partial x^{2}=\partial^{2} y / \partial t^{2}
$$

and the region of concern is the rectangle $R, 0 \leqq x \leqq 1,0 \leqq t \leqq \alpha$. There are solutions of the form $y=\sin (\pi n x) \sin (\pi n t)$ for any integer $n$. Then if $\alpha$ is a rational number, say $\alpha=m / n$, it is seen that given $y$ at $t=0$ and $t=\alpha$ does not determine $y$ at intermediate times. On the other hand, we found that if $\alpha$ was irrational and $y$ was of class $C^{2}$ in the rectangle $R$, then $y$ was uniquely determined.

An address delivered before the Annual Meeting in San Francisco on January 16, 1974 by invitation of the Committee to Select Hour Speakers for Annual and Summer Meetings; received by the editors March 11, 1974.

$A M S$ (MOS) subject classifications (1970). Primary $35 L 05,81 A 06,42 A 08,26 A 82$, $42 A 68,73 K 10,94 A 20,82 A 65,70 J 05,80 A 20,62 M 10,30 A 95,80 A 30$; Secondary $41 A 10$.

1 Prepared under Research Grant DA-ARO-D-31-124-71-G17.

Copyright (c) American Mathematical Society 1974 
Thus the irrationality of $\alpha$ established the uniqueness of the Dirichlet problem. To establish existence we had to assume that $\alpha$ could not be approximated too rapidly by rationals. For example suppose that for suitable positive constant $A$ and integer $k,|\alpha-m| n|>A| n^{k}$ for all integers $m$ and $n$. Thus if the values of $y$ at times $t=0, t=\alpha$ are functions of class $C^{3+k}$, then there exists a solution of the wave equation at intermediate times. It is of interest to note that the above inequality holds if $\alpha$ is an algebraic number of degree $k$.

This mathematical problem which we solved was inspired by the physical model of a vibrating string. However it is not clear what the mathematical solution really implies for the physical situation. Thus what is the physical significance of the statement that a time interval may be an irrational number but not a rational number?

1. D. G. Bourgin and R. J. Duffin, The Dirichlet problem for the vibrating string equation, Bull. Amer. Math. Soc. 45 (1939), 851-858. MR 1, 120.

2. Heavy Photon. Maxwell's equations give the wave equation for the photon. The photon has zero rest mass. Proca proposed the following modification of Maxwell's equations for a particle of rest mass $m$,

$$
\frac{\partial \varphi_{j}}{\partial x_{i}}-\frac{\partial \varphi_{i}}{\partial x_{j}}=\mu f_{i j}, \quad \sum_{1}^{4} \frac{\partial f_{i j}}{\partial x_{i}}=\mu \varphi_{j} .
$$

Here $\mu^{2}=-m^{2} c^{2}$ and $x_{4}^{2}=-c^{2} t^{2}$. These equations are somewhat unwieldy to manipulate. Taking a hint from Dirac, I found that the system of linear equations (1) may be expressed as a single vector wave equation

$$
\sum_{1}^{4} \beta_{i} \frac{\partial \psi}{\partial x_{i}}=\mu \psi
$$

Here $\psi$ is the column vector with ten components

$$
\psi=\left(\varphi_{1}, \varphi_{2}, \varphi_{3}, \varphi_{4}, f_{14}, f_{24}, f_{34}, f_{23}, f_{31}, f_{12}\right),
$$

and $\beta_{1}, \beta_{2}, \beta_{3}$, and $\beta_{4}$ denote 10 by 10 constant matrices. They were found to satisfy the following identities

$$
\beta_{i}^{3}=\beta_{i}, \quad \beta_{i} \beta_{j}^{2}+\beta_{j}^{2} \beta_{i}=\beta_{i}, \quad \beta_{i} \beta_{j} \beta_{k}+\beta_{k} \beta_{j} \beta_{i}=0 .
$$

These have since been termed the Duffin, Petiau, Kemmer commutation relations. Let

$$
L_{i j}=\left(x_{i} \frac{\partial}{\partial x_{j}}-x_{j} \frac{\partial}{\partial x_{i}}\right)-(-1)^{1 / 2} \hbar S_{i j},
$$

where $S_{i j}=\beta_{i} \beta_{j}-\beta_{j} \beta_{i}$. Then the angular momentum operator $L_{i j}$ is seen to commute with the operator of equation (2). Moreover the matrix 
$(-1)^{1 / 2} S_{i j}$ has eigenvalues 1,0 , and -1 . The physical interpretation of this is that the Proca particle has spin 1.

There are various other properties of the $\beta$ matrices: If the $\beta$ are transformed as components of a four vector, then the commutation relations are invariant under a Lorentz transformation. Again the $\beta$ generate an interesting semisimple algebra.

The conclusion to be drawn from this study is that the matrices implicit in a system of partial differential equations may give rise to an algebraic formalism both elegant and significant.

1. R. J. Duffin, On the characteristic matrices of covariant systems, Phys. Rev. 54 (1938), 1114.

2. - On wave equation vector-matrices and their spurs, Phys. Rev. (2) 77 (1950), 683-685. MR 11, 543.

3. Bernstein inequality for nonanalytic functions. The following theorem of S. Bernstein plays a central role in approximation theory.

A. Let $p(x)$ be a trigonometric polynomial of degree $n$,

$$
p(x)=\sum_{0}^{n}\left(a_{v} \cos v x+b_{v} \sin v x\right) .
$$

If $|p(x)| \leqq 1$ for all $x$, then $|d p| d x \mid \leqq n$.

It seemed desirable to extend this theorem to nonanalytic functions. To do this Albert Schaeffer and I proved the following theorem.

B. Let $f$ be a function such that for some integer $m$ and for all $x$,

$$
\{f(x)\}^{2} \leqq 1, \quad\left\{f^{(m)}(x)\right\}^{2}+\left\{f^{(m-1)}(x)\right\}^{2} \leqq 1 .
$$

Then for $k=1,2, \cdots, m$,

$$
\left\{f^{(k)}(x)\right\}^{2}+\left\{f^{(k-1)}(x)\right\}^{2} \leqq 1 .
$$

The proof begins by considering the case $m=2$. Consider the function

$$
\varphi(x)=\left\{f^{\prime}(x)\right\}^{2}+\{f(x)\}^{2} .
$$

If $\varphi(x) \leqq 1$ does not hold, then $\varphi$ has a local maximum at some point $x_{0}$ and $\varphi\left(x_{0}\right)>1$. Thus $\varphi^{\prime}\left(x_{0}\right)=2 f^{\prime}\left(x_{0}\right)\left\{f\left(x_{0}\right)+f^{\prime \prime}\left(x_{0}\right)\right\}=0$. If the first factor is zero, then $\varphi\left(x_{0}\right)=\left\{f\left(x_{0}\right)\right\}^{2} \leqq 1$. If the second factor is zero, then $\varphi\left(x_{0}\right)=\left\{-f^{\prime \prime}\left(x_{0}\right)\right\}^{2}+\left\{f^{\prime}\left(x_{0}\right)\right\}^{2} \leqq 1$. This contradiction proves the theorem in the case $m=2$. The proof is completed by induction.

Theorem B proves Bernstein's inequality. To see this let $f(x)=p(x / \lambda)$ where $\lambda$ is some constant greater than $n$. It follows that as $m \rightarrow \infty, f^{(m)}(x) \rightarrow$ 0 uniformly. Thus for $m$ sufficiently large, the conditions of Theorem B 
are satisfied so

$$
1 \geqq\left|f^{\prime}(x)\right|=\left|p^{\prime}(x / \lambda) / \lambda\right| .
$$

Allowing $\lambda \rightarrow n$ shows that $\left|p^{\prime}\right| \leqq n$. Q.E.D.

1. R. J. Duffin and A. C. Schaeffer, On the extension of a functional inequality of S. Bernstein to non-analytic functions, Bull. Amer. Math. Soc. 46 (1940), 356-363. MR 1, 205.

4. A refinement of Markoff's inequality. The following theorem of A. Markoff plays an important role in approximation theory.

Let $p(x)$ be a polynomial of degree $n$,

$$
p(x)=\sum_{0}^{n} a_{i} x^{i}
$$

If

(a)

$$
|p(x)| \leqq 1 \text { for }-1 \leqq x \leqq 1,
$$

then $\left|p^{\prime}(x)\right| \leqq n^{2}$ in the same interval.

Albert Schaeffer and I found a refinement of this theorem to the effect that the conclusion holds when (a) is replaced by the weaker condition

$$
|p(\cos k \pi / n)| \leqq 1 \text { for } k=0,1, \cdots, n .
$$

The proof employed rather involved complex variable techniques.

In recent years a more general approach to such problems has been developed by use of functional analysis. Is it possible, by such methods, to extend the refined Markoff inequality to a wider class of functions?

1. R. J. Duffin and A. C. Schaeffer, A refinement of an inequality of the Brothers Markoff, Trans. Amer. Math. Soc. 50 (1941), 517-528. MR 3, 235.

5. Representation of Fourier integrals as sums. Fourier integrals are an indispensible tool in almost all branches of applied mathematics. Since these integrals are difficult to evaluate numerically, I sought to replace the integrals by series.

Given an arbitrary function $\varphi(x)$, let functions $f(x)$ and $g(x)$ be defined by the sums:

$$
\begin{aligned}
& f(x)=\varphi(x)-(1 / 3) \varphi(x / 3)+(1 / 5) \varphi(x / 5)-\cdots, \\
& g(x)=(1 / x) \varphi(1 / x)-(1 / x) \varphi(3 / x)+(1 / x) \varphi(5 / x)-\cdots .
\end{aligned}
$$

Then $f(x)$ and $g(x)$ are Fourier sine transforms; that is,

$$
g(x)=\int_{0}^{\infty} \sin (\pi x t / 2) f(t) d t,
$$




$$
f(x)=\int_{0}^{\infty} \sin (\pi x t / 2) g(t) d t
$$

under mild restriction on $\varphi$.

An intuitive proof of this can be based on the well-known Poisson summation formula for the sine transform,

(3) $f(x)-f(3 x)+f(5 x)-\cdots=\frac{1}{x} g\left(\frac{1}{x}\right)-\frac{1}{x} g\left(\frac{3}{x}\right)+\frac{1}{x} g\left(\frac{5}{x}\right) \cdots$.

This holds for sine transform pairs $(f, g)$. If $(1 \mathrm{a})$ and $(1 \mathrm{~b})$ are substituted in (3), it is seen that both sides reduce to

$$
\sum_{1}^{\infty} \sum_{1}^{\infty} \frac{\alpha_{n} \alpha_{m}}{n} \varphi\left(\frac{m x}{n}\right)
$$

where $\alpha_{1}=1, \alpha_{2}=0, \alpha_{3}=-1$, etc. Since $x$ is arbitrary, this is an indication of the validity of (1) and (2).

The left side of (3) is a Möbius series $M(x)$,

$$
M(x)=f(x)-f(3 x)+f(5 x)-\cdots .
$$

The inversion of this series is

$$
f(x)=M(x)-\mu_{3} M(3 x)+\mu_{5} M(5 x)-\cdots,
$$

where $\mu_{n}$ is the well-known Möbius symbol. Applying this inversion to the right side of (3) gives

$$
f(x)=\sum_{n=1}^{\infty} \sum_{m=1}^{\infty} \frac{\mu_{n} \alpha_{n} \alpha_{m}}{n x} g\left(\frac{m}{n x}\right) .
$$

This is a direct representation of the Fourier sine transform as a double sum.

1. R. J. Duffin, Representation of Fourier integrals as sums. I, Bull. Amer. Math. Soc. 51 (1945), 447-455. MR 6, 266.

2. - Representation of Fourier integrals as sums. II, Proc. Amer. Math. Soc. 1 (1950), 250-255. MR 11, 592; MR 12, 1002.

3. - Representation of Fourier integrals as sums. III, Proc. Amer. Math. Soc. 8 (1957), 272-277. MR 18, 893.

4. H. F. Weinberger, Fourier transforms of Mobius series, Ph.D. Thesis, CarnegieMellon University, Pittsburgh, Pa., 1950.

6. Hadamard's conjecture on the clamped plate. The following conjecture was made by Hadamard in his 1908 prize memoir on the elastic plate.

(A) If a perpendicular force is applied at some point of a thin, flat, elastic plate which is rigidly clamped on its boundary then the displacement of the plate is of one sign at all points. 
Suppose that the plate is in the $(x, y)$ plane, then a displacement $w(x, y)$ of the plate satisfies the biharmonic equation

$$
\left(\partial^{2} / \partial x^{2}+\partial^{2} / \partial y^{2}\right)^{2} w=0
$$

in a region $R$ where no forces are applied. By an argument appealing to the reciprocity principle (conservation of energy), it may be shown that Hadamard's conjecture is equivalent to the following assertion.

(B) Suppose a function $w$ is biharmonic in a region $R$, and that on the boundary $w \geqq 0$ and $-\partial w / \partial n \geqq 0$, where $n$ is the exterior normal. Then $w \geqq 0$ throughout $R$. (This statement could be interpreted as a maximum principle for biharmonic functions.)

The assertion (B) is false, as the following counterexample shows. First it is observed that if $q$ is any complex constant, then $e^{i q x} \sinh q y$ and $e^{\imath q x} \cosh q y$ are both harmonic functions. Moreover if $h$ is a harmonic function, $y h$ is biharmonic. Thus

$$
f=e^{i q x}(-\cosh q y+y \operatorname{coth} q \sinh q y)
$$

is a biharmonic function. If $q=1.12+i 2.10$, then $q+\cosh q \sinh q=0$, and it is easy to check that $f=0$ and $\partial f / \partial y=0$ on the lines $y= \pm 1$. Let $w=\operatorname{Re}(f)$. Then $w(x, 0)=-e^{-2 \cdot 1 x} \cos 1.12 x$, so $f$ has nodal lines which intersect the $x$ axis at $1.12 x=\pi / 2+n \pi$. Let the region $R$ be bounded by the lines $y= \pm 1$ and the nodal lines which intersect the $x$ axis at $1.12 x=$ $\pm 3 \pi / 2$.

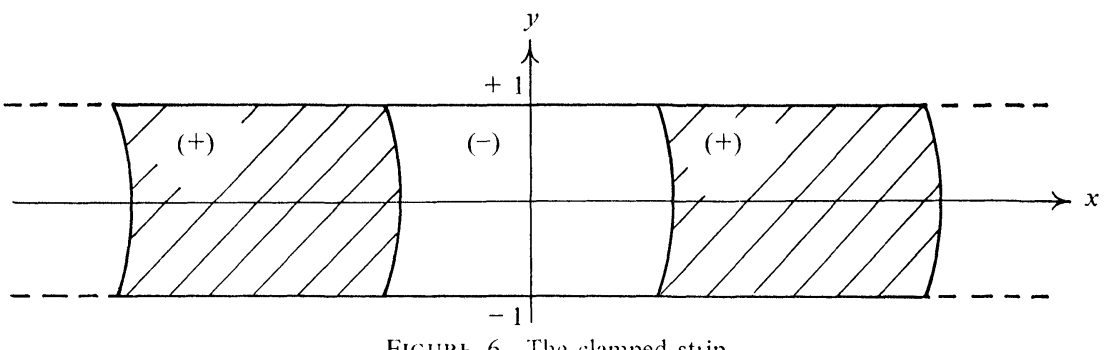

Figure 6. The clamped stıip

Then $R$ is divided into three congruent sections as is shown in the figure. Then $w \leqq 0$ in the central section and $w \geqq 0$ in the others. Thus $w=0$ and $-\partial w / \partial n \geqq 0$ on the boundary of $R$. However $w(0,0)<0$, and this contradicts statement (B). Hence statements (A) and (B) are both false.

The question still remained of whether or not there is any correct analog of the maximum principle for the biharmonic equation. The following is one such principle:

(C) Let $w$ be biharmonic in a region $R$, let $(a, b)$ be a point in $R$, and let 
$r$ be the vector with components $x-a, y-b$. Then

$$
w(a, b) \leqq \max _{\partial R}\left[w-r \cdot \nabla w+r^{2} \Delta w / 4\right]
$$

where $\partial R$ denotes the boundary of $R$.

Proof. A short calculation shows that if $w$ is biharmonic, then $w-r \cdot \nabla w+r^{2} \Delta w / 4$ is harmonic. Thus the inequality follows from the classical maximum principle.

Presumably such maximum principles for biharmonic functions could have applications in elasticity.

1. R. J. Duffin, On a question of Hadamard concerning super-biharmonic functions, J. Mathematical Phys. 27 (1949), 253-258. MR 10, 534.

2. - The maximum principle and biharmonic functions, J. Math. Anal. Appl. 3 (1961), 399-405. MR 26 \#1617.

7. Szegö's conjecture on the clamped plate. The normal modes of vibration of a clamped plate satisfy the biharmonic wave equation

$$
\Delta^{2} w=\lambda^{4} w .
$$

The boundary conditions are, of course,

$$
w=0, \quad \partial w / \partial n=0 .
$$

Szegö assumed that the gravest mode of vibration is free of nodal lines. Under this hypothesis he proved that of all plates of a given area, the circular plate has the gravest tone.

Studies made in collaboration with Alfred Schild and Douglas Shaffer indicate that Szegö's conjecture is not universally valid. In particular we solved equation (1) for a ring-shaped plate. In this geometry the wave equation is separable, and solutions have the form

$$
w=f_{n}(r) \cos n \theta .
$$

The radial part $f_{n}(r)$ is of the form

$$
f_{n}(r)=a J_{n}(\lambda r)+b Y_{n}(\lambda r)+c I_{n}(\lambda r)+d K_{n}(\lambda r),
$$

where $J_{n}, Y_{n}, I_{n}$, and $K_{n}$ are standard notation for the Bessel functions. The constants $a, b, c, d$ are chosen to satisfy the boundary condition (2) on both the inner and outer circles.

We found that if the outer circle had a diameter of over 715 times the diameter of the inner circle, then the gravest mode of vibration has a diametral nodal line. This corresponds to $n=1$ in equation (3). We also found the conjecture to be false in a simply connected domain.

1. G. Szegö, On membranes and plates, Proc. Nat. Acad. Sci. U.S.A. 36 (1950), 210-216. MR 11, 757. 
2. R. J. Duffin and D. H. Schaffer, On the modes of vibration of a ring-shaped plate, Bull. Amer. Math. Soc. 58 (1952), 652.

3. R. J. Duffin, Nodal lines of a vibrating plate, J. Mathematical Phys. 31 (1953), 294-299. MR 14, 601.

4. R. J. Duffin and A. Schild, The effect of small constraints on natural vibrations, Proc. Sympos. Appl. Math., vol. 5., McGraw-Hill, New York, 1954, pp. 155-163.

5. - On the change of natural frequencies induced by small constraints, J. Math. Mech. 6 (1957), 731-758. MR 19, 1101.

8. The Wang algebra of networks. Consider a network of electrical conductors such as shown in the figure. To determine the joint conductance of the network, one could set up Kirchhoff's equations and solve for the current flow through the battery.

K. T. Wang managed an electrical power plant in China, and in his spare time sought simple rules for solving the network equations. Wang's rules were published in the reference indicated below [5]. Wang could not write in English so his paper was actually written by his son, then a college student. Raoul Bott and I recognized that Wang's rules actually define an algebra. We restated the rules as three postulates for an algebra:

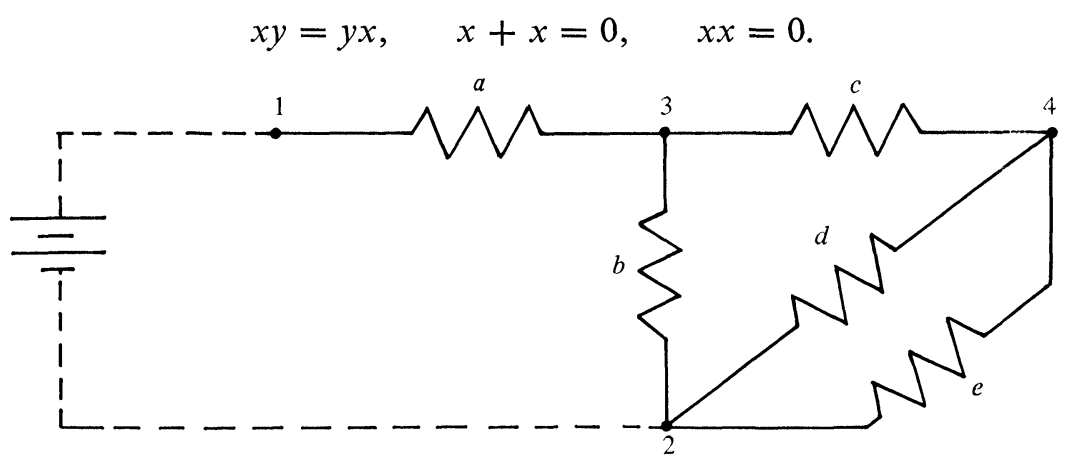

Figure 8. A simple network

To apply the Wang algebra to the network shown, let the conductances of the various branches be $a, b, c, d$ and $e$. Also regard these symbols as independent generators of a Wang algebra. A star element of the algebra is defined as the sum of the branches meeting at a node. Thus the star element at node 3 is $a+b+c$.

An algorithm for finding the joint conductance between nodes 1 and 2 follows. First form $P$, the Wang product of all star elements except those at nodes 1 and 2 . Thus

$$
P=(a+b+c)(c+d+e) .
$$

Using the postulate (iii) gives

$$
P=a c+a d+a e+b c+b d+b e+c d+c e .
$$


Next form the Wang product $T$ of all stars except one. Then

$$
T=a P=a b c+a b d+a b e+a c d+a c e . \text { (All trees!) }
$$

Then the joint conductance $K$ between nodes 1 and 2 is given as the ratio

$$
K=\frac{T}{P}=\frac{a b c+a b d+a b e+a c d+a c e}{a c+a d+a e+b c+b d+b e+c d+c e} .
$$

The Wang algebra has interesting and important connections with matroid theory, totally unimodular matrices, and Grassmann algebra. In fact Wang algebra is Grassmann algebra over the mod 2 field. The trees of any graph are given by the above algorithm for $T$.

1. R. Bott and R. J. Duffin, On the Wang algebra of networks, Bull. Amer. Math. Soc. 57 (1951), 136.

2. - On the algebra of networks, Trans. Amer. Math. Soc. 74 (1953), 99-109. MR 15, 95 .

3. R. J. Duffin, An analysis of the Wang algebra of networks, Trans. Amer. Math. Soc. 93 (1959), 114-131. MR 22 \#49.

4. — Network models, Mathematical Aspects of Electrical Network Theory, SIAM-AMS Proc., vol. 3, Amer. Math. Soc., Providence, R.I., 1971, pp. 65-91.

5. K. T. Wang, On a new method of analysis of electrical networks, Memoir 2, National Research Institute of Engineering, Academia Sinica, 1934.

9. Sampling of particle size by planar sectioning. In some aluminumsilicon alloys, most of the silicon is distributed as small particles throughout an aluminum matrix. It is of importance in metallurgy to know the distribution of particle size. However it is very difficult to observe directly the size distribution. Instead, linear or planar samples are observed from a cross section of the alloy as shown in the figure. The problem thereby posed is the determination of the true size distribution of the particle from the observation of linear or planar samples.

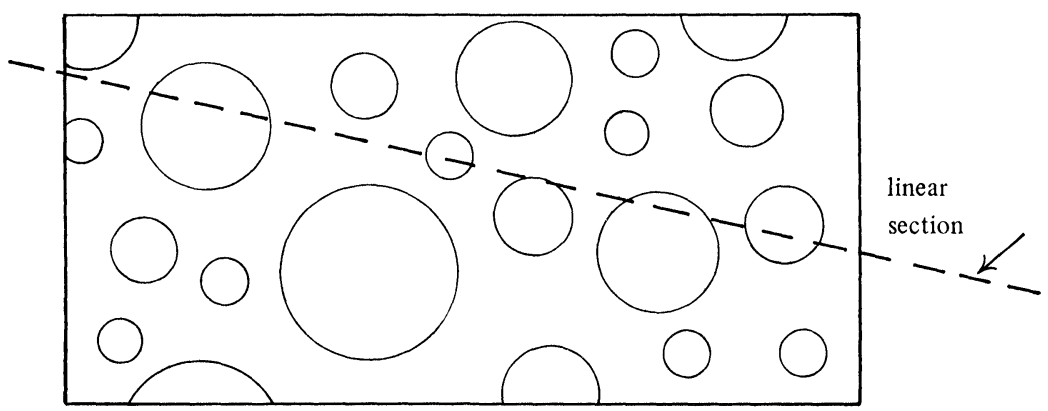

FIGURE 9. A planar section of particles 
In a study made in collaboration with Russell Meussner and Frederick Rhines, it was found that if the particles are assumed to be spherical, then the size distribution of linear or planar sections is related to the true size distributions by certain integral equations. These integral equations are analogous to Abel's equation and the fractional integration of Herman Weyl [1] and it proved possible to obtain the resolvent kernels.

The following distribution functions were related:

(a) The sphere distribution function $G_{3}(s)$ is the average number of spheres per cubic centimeter having diameter greater than $s$.

(b) The circle distribution function $G_{2}(s)$ is the average number of circles per square centimeter having diameter greater than $s$. The circles are the intersection of a plane with the spherical particles.

(c) The segment distribution function $G_{1}(s)$ is the average number of segments per centimeter having length greater than $s$. The segments are the intersection of a line with the spherical particles.

THEOREM 1. The cumulative distribution functions are related by the following Stieltjes integrals:

$$
\begin{aligned}
& G_{2}(s)=-\int_{s}^{\infty}\left(n^{2}-s^{2}\right)^{1 / 2} d G_{3}(n), \\
& G_{3}(s)=-\frac{2}{\pi} \int_{s}^{\infty}\left(n^{2}-s^{2}\right)^{-1 / 2} d G_{2}(n), \\
& G_{1}(s)=-\frac{\pi}{4} \int_{s}^{\infty}\left(n^{2}-s^{2}\right) d G_{3}(n), \\
& G_{3}(s)=-(2 / \pi s) d G_{1} / d s, \\
& G_{1}(s)=-\int_{s}^{\infty}\left(n^{2}-s^{2}\right)^{1 / 2} d G_{2}(n), \\
& G_{2}(s)=-\frac{2}{\pi} \int_{s}^{\infty}\left(n^{2}-s^{2}\right)^{-1 / 2} d G_{1}(n) .
\end{aligned}
$$

A distribution function may be termed Gaussian if it has the form $G(s)=A \exp \left(-k s^{2}\right)$, where $A$ and $k$ are constants. (This is not the same as the Gaussian density function.)

THEOREM 2. If any two of the distribution functions $G_{1}, G_{2}$, and $G_{3}$ are proportional, then they are all Gaussian. If any one of them is Gaussian, all are Gaussian.

The proof of Theorem 2 follows from the relations of Theorem 1.

1. G. H. Hardy, J. E. Littlewood and G. Pólya, Inequalities, Cambridge, 1934, pp. 245, 290. 
2. R. A. Meussner, The growth of silicon particles in an aluminum matrix during isothermal heat treatment, Thesis, Carnegie-Mellon University, Pittsburgh, Pa., 1952.

3. R. J. Duffin, R. A. Meussner and F. N. Rhines, Statistics of particle measurement and of particle growth, Carnegie-Mellon University, Technical Report 32, April 1953.

10. Rayleigh quotient for dissipative systems. Consider a mechanical system of $n$-degrees of freedom vibrating about a position of static equilibrium. If the vibrations are small then Newton's equation can be written in the linear form

$$
A\left(d^{2} Q / d t^{2}\right)+C Q=0,
$$

where $A$ and $C$ are $n$ by $n$ symmetric positive definite matrices of constants. A normal mode of motion is of the form $Q=q e^{i w t}$, where the vector $q$ is independent of the time, and $w$ is the frequency of vibration. Thus $w^{2} A q-C q=0$. The Rayleigh quotient is

$$
R=[c(v) / a(v)]^{1 / 2},
$$

where $a$ and $c$ are the quadratic forms $(A v, v)$ and $(C v, v)$. The minimum frequency satisfies the relation

$$
w=\min _{v}[a(v) / c(v)]^{1 / 2}
$$

for arbitrary real vectors $v$. The maximum frequency satisfies a similar relation.

When frictional forces are introduced, Newton's equations take the form

$$
A \frac{d^{2} Q}{d t^{2}}+B \frac{d Q}{d t}+C Q=0
$$

where $B$ is also a symmetric positive definite matrix. Again let $a(v)$, $b(v)$, and $c(v)$ denote the corresponding quadratic forms. A solution of the form $q e^{-k t}$ satisfies the relation $k^{2} a(q)-k b(q)+c(q)=0$. To make sure that $k$ was real, I defined an overdamped system by the relation

$$
b^{2}(v)-4 a(v) c(v)>0
$$

for arbitrary real vectors $v$. The nonlinear eigenvalue $k$ corresponds to a motion of exponential decay (like radioactivity). It was found that the correspondent of the Rayleigh quotient is the functional

$$
R(v)=\left(b \pm\left(b^{2}-4 a c\right)^{1 / 2}\right) / 2 a .
$$

Then the largest decay constant is given by

$$
k=\max _{v}\left(\frac{b+\left(b^{2}-4 a c\right)^{1 / 2}}{2 a}\right) .
$$


The smallest decay constant satisfies

$$
k=\min _{v}\left(\frac{b-\left(b^{2}-4 a c\right)^{1 / 2}}{2 a}\right) .
$$

A similar theory holds when the frictional forces are replaced by gyroscopic forces. Then the matrix $B$ is skew symmetric.

1. R. J. Duffin, A minimax theory for overdamped networks, J. Rational Mech. Anal. 4 (1955), 221-233. MR 16, 979.

2. - The Rayleigh-Ritz method for dissipative or gyroscopic systems, Quart. Appl. Math. 18 (1960/61), 215-221. MR 22 \#12775.

11. Optimization of cooling fins. A common problem of heat transfer is the design of machinery so that the structure can dissipate excess heat. For example cooling fins are used on cylinders of air-cooled engines as

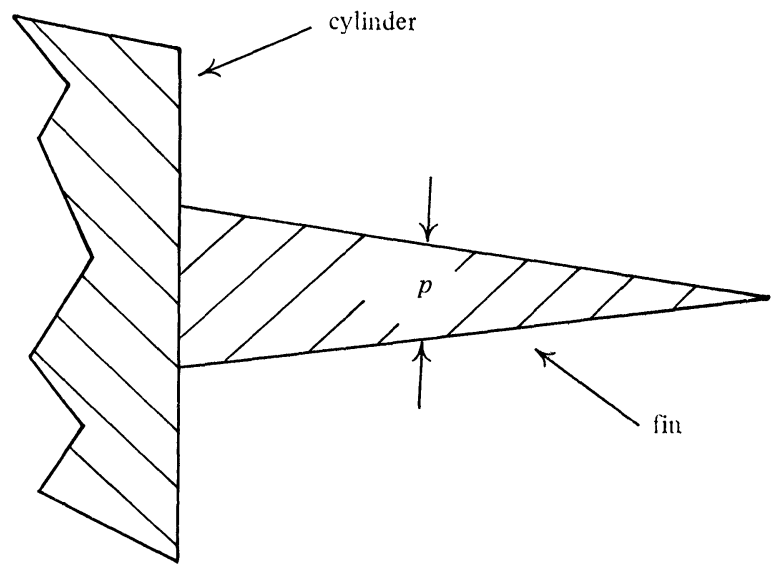

FIGURE 11. Cooling fin cross section

shown in the figure. Suppose for such an example that the fin is not permitted to exceed a given weight. It is not difficult to see that the fin should taper, narrowing in the direction of heat flow. The optimum design problem arising may be phrased in this way-find the taper, a thickness function $p(x)$, which gives the maximum dissipation of heat for a given weight of fin.

If the fin is of constant width, the weight of the fin is proportional to $\int_{0}^{L} p(x) d x$, where $L$ is the length of the fin. The heat dissipated to the air is proportional to $\int_{0}^{L} u(x) d x$, where $u(x)$ is the temperature of the fin relative to the air. Of course, $u$ has a prescribed value $u_{0}$ on the cylinder, 
$x=0$. If the fin is thin the equation of heat conduction has the form

$$
\frac{d}{d x}\left(p(x) \frac{d u}{d x}\right)=c u
$$

where $c$ is constant.

Conceivably this problem could be treated by solving the differential equation for various choices of $p$ and $L$ and retaining the best solution. Such an approach would be very difficult to implement. Instead the differential equation was regarded as the Euler equations of a variational problem. Then a grand variational problem was set up in which both the temperature function $u$ and the taper function $p$ were variables. The Euler equation for this grand variational problem proved to be very simple. The condition on the temperature was simply that the temperature gradient should have constant magnitude at all points of the fin. This relation greatly simplifies the equation of heat conduction. It then results that the taper $p$ is obtained by quadrature and the design problem is completely solved.

The above condition on the gradient was first obtained for the above special geometry. Later more general models were used and the same criterion was found to hold. The gradient condition might be stated as a "democracy" criterion thus: To obtain the maximum dissipation of heat from a cooling fin of a given weight, the fin should be so proportioned that each part of the fin carries the same heat current.

1. R. J. Duffin, A variational problem relating to cooling fins, J. Math. Mech. 8 (1959), 47-56. MR 21 \#2477.

2. R. J. Duffin and D. K. McLain, Optimum shape of a cooling fin on a convex cylinder, J. Math. Mech. 17 (1968), 769-784.

3. R. J. Duffin, Optimum heat transfer and network programming, J. Math. Mech. 17 (1968), 759-768.

4. S. Bhargava and R. J. Duffin, Network models for maximization of heat transfer under weight constraints, Networks 2 (1972), 355-365.

5. - Dual extremum principles relating to cooling fins, Quart. J. Appl. Math. 31 (1973), 27-41.

12. Prediction algorithms based on discounted least squares. In applied science it is often required to make extrapolations into the future based on observations obtained at regular time intervals in the past. A standard approach to such a problem is to extrapolate with a polynomial fitted to the data by least squares.

Theodore Schmidt and I were presented with such a situation where the observations of the distant past should be given less weight than those more recent. This discounting can be achieved by use of an exponential weight factor. Thus suppose that $y_{1}, y_{2}, y_{3}, \cdots$ is a sequence of real numbers giving the observation at previous times $x=1,2,3, \cdots$. The central 
problem is to predict $y_{0}$, the value at $x=0$. Let $p(x)$ be a polynomial, say

$$
p(x)=a+b x+c x^{2} .
$$

Then the coefficients are chosen to minimize

$$
E=\sum_{n=1}^{\infty} \theta^{n}\left[y_{n}-p(n)\right]^{2} .
$$

Here the constant $\theta<1$ is the discount factor. Then the predicted value of $y_{0}$ is defined as $y_{0}^{*}=p(0)$.

When the minimization is carried out, a "long formula" of the following type results:

$$
y_{0}^{*}=\sum_{n=1}^{\infty} Q_{n} y_{n} .
$$

Here the constants $Q_{n}$ can be obtained explicitly. However there is a much better algorithm termed "the short formula" given as

$$
y_{0}^{*}=3\left(y_{1}+\theta \delta_{1}\right)-3\left(y_{2}+\theta^{2} \delta_{2}\right)+\left(y_{3}+\theta^{3} \delta_{3}\right) .
$$

Here $\delta_{k}=y_{k}^{*}-y_{k}$, where $y_{k}^{*}$ is the predicted value based on the previous values $y_{k+1}, y_{k+2}, \cdots$. Thus the short formula gives the predicted value of $y$ in terms of the last three observed values and last three predicted values.

More generally if $p(x)$ is a polynomial of degree $m-1$, then the short formula is

$$
y_{k}^{*}=\sum_{j=1}^{m}(-1)^{j+1}\left(\begin{array}{c}
m \\
j
\end{array}\right)\left[y_{j+k}+\theta^{j} \delta_{j+k}\right] .
$$

Here $\left(\begin{array}{c}m \\ j\end{array}\right)$ is the binomial coefficient.

For some applications it seemed desirable to use trigonometric polynomials or exponential polynomials instead of algebraic polynomials. Then it again proves possible to have a short formula

$$
y_{k}^{*}=-\sum_{j=1}^{m}\left(g_{j} y_{j+k}+f_{j} \theta^{j} \delta_{j+k}\right) .
$$

Here the coefficients $g_{j}$ and $f_{j}$ are given by explicit formulae involving the polynomial terms.

1. R. J. Duffin and T. W. Schmidt, Simple formula for prediction and automatic scrutation, J. Amer. Rocket Soc. 30 (1960), 364-365.

2. R. J. Duffin, Discounted least squares, Proc. Fourth Ordnance Conference on Operations Research, Army Research Office, Durham, N.C., 1960.

3. R. J. Duffin and T. W. Schmidt, An extrapolator and scrutator, J. Math. Anal. Appl. 1 (1960), 215-227. MR 22 \#11510.

4. R. J. Duffin and P. Whidden, An exponomial extrapolator, J. Math. Anal. Appl. 3 (1961), 526-536. MR 36 \#2301. 
5. R. J. Duffin, Extrapolating time series by discounted least squares, J. Math. Anal. Appl. 20 (1967), 325-341. MR 36 \#1078.

13. Convolution products. The points of the complex plane with integer coordinates form a lattice which breaks up the plane into unit squares. A function $f$ is said to be discrete analytic on one of these squares if the difference quotient across one diagonal is equal to the difference quotient across the other diagonal

$$
\frac{f(z+1+i)-f(z)}{i+1}=\frac{f(z+i)-f(z+1)}{i-1} .
$$

This definition was introduced by Rufus Issacs and Jacqueline Ferrand (Lelong). This definition leads directly to difference equation analogs of the Cauchy-Riemann equations and the Laplace equation. Many of the theorems of continuous function theory can be extended to this discrete function theory.

If $f(z)$ and $g(z)$ are two analytic functions, then their product $f(z) g(z)$ is also an analytic function. This important property does not seem to carry over to discrete function theory in any simple way. However there is another type of product in the classical theory termed the convolution and defined as

$$
f(z) * h(z)=\int_{0}^{z} f(z-w) g(w) d w .
$$

The integration is performed along any contour connecting 0 and $z$. Charles Duris and I found that the same formula gives a product of two discrete analytic functions if the integral is interpreted in the following way. The contours are restricted to the lattice lines. Thus a contour is made up of unit line segments. The value of a discrete function at a point of the line segment is defined to be the average of the values at the end points. Thus the convolution product becomes the sum

$$
f * g=\sum_{n=1}^{m} \frac{\left[f\left(z-z_{n}\right)+f\left(z-z_{n-1}\right)\right]}{2} \frac{\left[g\left(z_{n}\right)+g\left(z_{n-1}\right)\right]}{2}\left(z_{n}-z_{n-1}\right),
$$

where $0=z_{0}, z_{1}, z_{2}, \cdots, z_{m}=z$ is a chain of lattice points.

The convolution product of discrete analytic functions is again a discrete analytic function. The product is both commutative and associative. Thus the convolution product yields an algebra for discrete function theory.

These algorithms can be extended to arbitrary partial difference equations with constant coefficients in the plane. With each such partial differential equation, Joan Rohrer and I associated a convolution product. 
Given any two solutions of the partial difference equation, the convolution product is again a solution.

1. R. J. Duffin and C. S. Duris, A convolution product for discrete function theory, Duke Math. J. 31 (1964), 199-220. MR 29 \#429.

2. R. J. Duffin and Joan Rohrer, A convolution product for the solutions of partial difference equations, Duke Math. J. 35 (1968), 683-698. MR 39 \#1831.

14. Convexity and chemical equilibrium. Consider a mixture of gases in a reaction chamber and suppose that the temperature $T$ and pressure $P$ are maintained constant. Then according to Gibbs, the mixture is in equilibrium if the free energy $G$ is a minimum. For example consider the chemical reaction

$$
2 \mathrm{H}_{2}+\mathrm{O}_{2}=2 \mathrm{H}_{2} \mathrm{O} \text { (steam). }
$$

Let $x_{1}$ be the number of molecules of $\mathrm{H}_{2}, x_{2}$ be the number of molecules of $\mathrm{O}_{2}$, and $x_{3}$ be the number of molecules of $\mathrm{H}_{2} \mathrm{O}$. If it is assumed that there is only a negligible number of other types of molecules present, then $G=G\left(x_{1}, x_{2}, x_{3}\right)$. This function is to be minimized subject to the mass balance constraints

$$
2 x_{1}+2 x_{3}=e_{1}, \quad 2 x_{2}+x_{3}=e_{2} .
$$

Here $e_{1}$ is the total number of hydrogen atoms in the chamber, and $e_{2}$ is the total number of atoms of oxygen in the chamber. Thus the equilibrium state is obtained by minimizing $G$ subject to the mass balance constraints.

To obtain the form of the free energy function $G$, chemists assume the perfect gas laws of Boyle, Charles, and Dalton. Then $G$ is given as a definite logarithmic formula involving constants which chemists determine experimentally. Moreover, it is found that $G(x)$ so evaluated is a convex function. Thus, in modern terminology, the Gibbs procedure is termed a convex program.

As is well known, each convex program has a dual program concerning the maximization of a concave function $G^{*}$. Then the theory of such programs gives the duality inequality

$$
G\left(x_{1}, x_{2}, x_{3}\right) \geqq M \geqq G^{*}\left(y_{1}, y_{2}\right) .
$$

Here $M$ is the minimum of the primal program and also the maximum of the dual program. The dual variable $y_{1}$ is interpolated as the chemical potential of atomic hydrogen, i.e. $y_{1}=\partial G / \partial e_{1}$. Likewise $y_{2}$ is the chemical potential of atomic oxygen.

The same mathematical theory often applies to entirely different physical situations. Nevertheless it was somewhat surprising when Avriel, Passy, and Wilde pointed out that the above described duality theory of 
chemical equilibrium of perfect gases is essentially identical with the duality theory of geometric programming. Geometric programming concerns the economics of engineering design. The basic problem is to minimize the cost of construction and operation of a device or system.

Of course the assumption that the various compounds in a chemical reaction obey perfect gas laws is at best an approximation. Zener and I wondered whether or not the duality inequality is just an approximation. By making some mild assumption of a physical nature, we were able to show the true Gibbs function is convex, and that there exists a concave function $G^{*}$ which satisfies the duality inequality (3). We termed $G^{*}$ the anti-Gibbs function.

1. R. J. Duffin, E. L. Peterson and C. Zener, Geometric programming: Theory and application, Wiley, New York, 1967. MR 35 \#5225.

2. R. J. Duffin and C. Zener, Geometric programming, chemical equilibrium and the anti-entropy function, Proc. Nat. Acad. Sci. U.S.A. 63 (1969), 629-636.

3. - Geometric programming and the Darwin-Fowler method in statistical mechanics, J. Chem. Phys. 74 (1970), 2419-2423.

4. R. J. Duffin, Duality inequalities of mathematics and science, Nonlinear Programming (Proc. Sympos., Univ. of Wisconsin, Madison, Wis., 1970), edited by J. B. Rosen, O. L. Mangasarian and K. Ritter, Academic Press, New York, 1970, pp. 401-423.

15. Associative network operations. Kirchhoff defined a network as an interconnection of resistors at nodal points. For the present purpose a network may be regarded as a black box. The nodes of the network on the surface of the box are called terminals and are ordered $1,2, \cdots, n$. The network may or may not have other nodes inside the box. William Anderson, George Trapp and I have studied the problem of the interconnection of such network boxes to form larger networks. Thereby we have been led to some interesting algebraic questions.

Consider two network boxes $R$ and $S$, each having $n$ terminals. How can $R$ and $S$ be conjoined to form a larger network $X$ also having $n$ terminals ? As a first approach to this question we defined a device termed a junctor. A junctor is a $3 n$-terminal network box. The terminals are separated into three equal banks $A, B$, and $J . A$ and $B$ are termed input banks, and $J$ is termed the output bank. Inside the junctor box the terminals are interconnected by wires of zero resistance. Such connections are subject to certain mild restrictions to ensure desirable physical properties. Thus unconnected terminals or short circuits are not allowed.

The two network boxes are $R$ and $S$, and are "plugged" into the input banks $A$ and $B$ of the junctor. Then the output bank $J$ forms the terminals of a new $n$-terminal network $X$. Thus interconnections can be symbolized as

$$
X=J(R, S) .
$$


A junctor can be diagrammed as a triangular box. Thus Figure 15 shows a junctor for interconnecting 4 terminal networks termed the hybrid junctor.

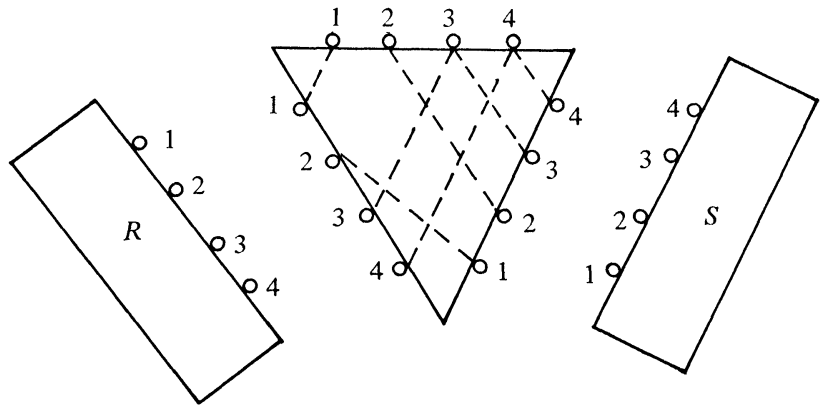

FIGURE 15. The hybrid junctor

The dotted lines denote the internal connections.

Let $T$ be another $n$-terminal network box and suppose that there are two identical junctors $J$. Then a new $n$-terminal box $Y$ can be constructed as

$$
Y=J(X, T)=J(J(R, S), T) .
$$

This raises the question of the associativity of the junctor operations. Thus, is

$$
J(J(R, S), T)=J(R, J(S, T)) ?
$$

The equality here is interpreted to mean that the interconnection between $R, S, T$ on the left and right are electrically equivalent. It is not difficult to show graphically that the hybrid junctor has the associativity property.

To study the general associativity problem we introduced $3 n$ by $3 n$ adjacency matrices for a junctor. The matrix elements are 0 or 1 . Thus 1 means an internal connection and 0 means no connection. Our study led to simple necessary and sufficient conditions to insure the associativity condition.

1. W. N. Anderson, Jr. and R. J. Duffin, Series and parallel addition of matrices, J. Math. Anal. Appl. 26 (1969), 576-594. MR 39 \#3904.

2. R. J. Duffin, Network models, Mathematical Aspects of Electrical Network Theory, SIAM-AMS Proc., vol. 3, Amer. Math. Soc., Providence, R.I., 1971, pp. 65-91.

3. R. J. Duffin and G. E. Trapp, Hybrid addition of matrices-A network theory concept, Carnegie-Mellon University Mathematics Report 70-44, November 1970; Applicable Anal. 2 (1972), 241-254.

4. W. N. Anderson, Jr., R. J. Duffin and G. E. Trapp, Tripartite graphs to analyze the interconnection of networks, Graph Theory and Applications, edited by Y. Alavi, D. R. Lick and A. T. White, Springer-Verlag, Berlin, 1972, pp. 7-12. 